\title{
Cross-sectional study about impact of parental smoking on rhinitis symptoms in children
}

\author{
A. Faraldo-García ${ }^{1}$, A. López-Silvarrey ${ }^{2}$, S. Pertega ${ }^{3}$, M.-J. Cruz ${ }^{4,5}$, M. Sampedro ${ }^{6}$, J. \\ Sánchez-Lastres ${ }^{6}$, M.A. San-José González ${ }^{6}$, L. Bamonde ${ }^{6}$, L. Garnelo ${ }^{6}$, T. Pérez- \\ Castro $^{7}$, L. Valdés-Cuadrado ${ }^{8,9,10}$, F.-J. González-Barcala ${ }^{4,8,9,10}$ \\ ${ }^{1}$ Departament of Otorhinolaryngology, University Hospital of Santiago de Compostela, Santiago de Compostela, \\ Spain \\ ${ }^{2}$ María José Jove Foundation, A Coruña, Spain \\ ${ }^{3}$ Clinical Epidemiology and Biostatistics Unit, University Hospital Complex, A Coruña, Spain \\ ${ }_{5}^{4}$ Spanish Biomedical Research Networking Centre-CIBERES, Madrid, Spain \\ ${ }^{5}$ Department of Respiratory Medicine, Hospital Universitari Vall d'Hebron, Barcelona, Spain \\ ${ }^{6}$ Departament of Pediatrics, Servicio Galego de Saúde, A Coruña, Spain \\ ${ }^{7}$ Grupo de investigación Cardiovascular (GRINCAR), Instituto de Investigación Biomédica de A Coruña (INIBIC), \\ Instituto Universitario de Ciencias de la Salud, Universidade da Coruña, A Coruña, Spain \\ ${ }^{8}$ Department of Medicine, University of Santiago de Compostela, Santiago de Compostela, Spain \\ ${ }^{9}$ Department of Respiratory Medicine, University Hospital of Santiago de Compostela, Santiago de Compostela, \\ Spain \\ ${ }^{10}$ Health Research Institute of Santiago de Compostela (IDIS), Santiago de Compostela, Spain
}

\begin{abstract}
Objective. Assess the prevalence of rhinitis and exposure to environmental tobacco smoke (ETS) of children in our community and its relationship with symptoms of rhinitis

Methods (design, setting, participants, main outcome measures). Cross-sectional study using questionnaire on rhinitis of the International Study of Asthma and Allergies in Childhood, in children (6-7 years) and adolescents (1314 years). Categories: "rhinitis ever", "recent rhinitis", "recent rhinoconjunctivitis", "severe rhinoconjunctivitis". Parental smoking: (i) neither parent smokes; (ii) only the mother smokes; (iii) only the father smokes; and (iv) both parents smoke. Odds ratio of the prevalence of symptoms of rhinitis according to ETS exposure was calculated using logistic regression.

Results. 10690 children and 10730 adolescents. The prevalence of "rhinitis ever" in children: $29.4 \%$, "recent rhinitis" $24 \%$, "recent rhinoconjunctivitis" $11.5 \%$ and "severe rhinoconjunctivitis" $0.1 \%$. In adolescents: $46.2 \%$, $34.5 \%, 16.2 \%$ and $0.2 \%$, respectively. Environmental tobacco smoke exposure in the home occurred in $51 \%$ of cases. Parental smoking was associated with a higher prevalence of forms of rhinitis in adolescents when only the mother was a smoker. In children when both parents were smokers.

Conclusion. Rhinitis is highly prevalent in our community. Environmental tobacco smoke exposure is still very common. The relationship between ETS and rhinitis symptoms in children of this community is not as robust as that found for asthma.
\end{abstract}

\section{Keypoints}

Rhinitis in children; Impact of parental smoking; Prevalence of rhinitis; Rhinitis symptoms in children. 


\section{INTRODUCTION}

Rhinitis is highly prevalent in childhood, especially in our population. ${ }^{1}$ It appears to have increased significantly in recent years. ${ }^{2,3}$ Both asthma and rhinitis are underdiagnosed and undertreated in both children and adults. A recent study involving a Danish population aged 14-44 years demonstrated underdiagnosis in $50 \%$ of asthmatics and in $32 \%$ of patients with rhinitis, with rates of undertreatment reaching $50 \%$ and $83 \%$, respectively. ${ }^{4}$

Environmental tobacco smoke (ETS) is the main domestic environmental pollutant. During the combustion of tobacco, over 8000 compounds are generated, including numerous toxic chemicals and irritants with acute adverse effects on health, in addition to human carcinogens. ${ }^{5}$ Passive exposure to tobacco smoke is common, and its harmful effects on health have been well-known for decades. ${ }^{6}$ However, the magnitude of the problem worldwide is not yet fully understood. ${ }^{7}$ There are multiple studies showing that exposure to tobacco smoke during childhood is associated with an increased risk of asthma. ${ }^{8,}$ In the case of rhinitis, fewer studies have been published and the results do not seem conclusive. Thacher et al. and Mitchell et al. find that exposure to second hand smoke during infancy was associated with an overall elevated risk rhinitis, while Lee et al. find that foetal exposure to maternal active smoking was significantly associated with asthma ever, but not with allergic rhinitis ever or eczema ever. $8,10,11$ Certain studies even seem to indicate a protective effect of ETS on the prevalence of rhinitis. $^{12}$

The aim of this study was to evaluate the prevalence of the rhinitis and the impact of parental smoking on rhinitis symptoms in children (6-7 years) and adolescents (13-14 years) in our community.

\section{MATERIALS AND METHODS}

A cross-sectional study was conducted using the method outlined in the ISAAC study (International Study of Asthma and Allergies in Childhood). ETS was assessed in children and adolescents residing in the Autonomous Community of Galicia (Spain), using the ISAAC questionnaire translated into Spanish and validated previously. ${ }^{1}$

The study was conducted in schools located in six of the seven health areas in the region, serving $69 \%$ of the total population (1.9 million). Two age groups, one for children aged 6-7 years and another for adolescents aged 13-14 years, were studied. Our sample is representative of the population of our region with regard to city, urban, countryside. Schools that declined to participate were replaced with other centres. Fieldwork was conducted between October 2006 and February 2007.

Authorization was obtained from the parents or legal guardians, who responded to the questionnaire for the 6 to 7-year-olds, while the adolescents aged 13-14 years completed the questionnaire themselves.

The questionnaire data were manually entered into a database in accordance with ISAAC protocols, using double entry with subsequent validation.

The questionnaire included questions about rhinitis symptoms, weight, height, presence of a dog or a cat in the home, parental smoking habits and the mother's educational level, mother occupation are not collected. Also included questions about asthma and dermatitis, but these diseases are treated in other publications. 
The standardised questionnaire on rhinitis symptoms includes the following six questions:

1. you (has your child) ever had a problem with sneezing or a runny or blocked nose, when you (he or she) DID NOT have a cold or "the flu"? (YES/NO)

2. In the past 12 months, have you (has your child) had a problem with sneezing or a runny or blocked nose, when you (he or she) DID NOT have a cold or "the flu"? (YES/NO)

3. the past 12 months, has this nose problem been accompanied by itchy-watery eyes? (YES/NO)

4. In which of the past 12 months did this nose problem occur? (Month names listed).

5. In the past 12 months, how much did this nose problem interfere with your (child's) daily activities? (Not at all/a little/ a moderate amount/a lot).

6. Have you (has your child) ever had hay fever? (YES/NO)

Based on the answers to the above questions, the following categories were defined:

1. "Rhinitis ever" determined by a positive response to question 1 .

2. "Recent rhinitis" determined by a positive response to question 2 .

3. "Recent rhinoconjunctivitis" determined by a combination of positive responses to questions 2 and 3.

4. "Severe rhinoconjunctivitis" determined by a positive response to questions 2 and 3, with the answer "a lot" to question $5 .{ }^{13}$

Based on the responses to the questionnaire, parental smoking was classified into four mutually exclusive categories: (i) no parent smoked; (ii) only the mother smoked; (iii) only the father smoked; (iv) both parents smoked. A category for ex-smokers and parameter of smoke inside the house or outside has not been collected because there are not included on the criteria of ISAAC study.

Obesity and overweight were defined according to body mass index (BMI) cutoffs established by Cole et al., ${ }^{14}$ for each age group and sex.

The educational level of the mother was classified into three categories: (i) no formal education or only primary education; (ii) secondary education; (iii) university education.

\subsection{Ethical considerations}

The study was approved by the Research Ethics Committee of Galicia.

\subsection{Statistical analysis}

For the statistical analysis, multiple logistic regression was used to obtain prevalence-adjusted odds ratio and $95 \%$ confidence intervals between rhinitis symptoms of school age children and parental smoking. Children of non-smoking parents comprised the control group.

In the multivariate analysis, the results were adjusted for gender, body mass index, maternal educational level and exposure to dogs and/or cats in the home.

Statistical analysis was performed using the spss 17.0. (SPSS Inc., Chicago, IL, USA) 


\section{RESULTS}

The response rate in the age group 6-7 years was 72.4\%, with 10690 valid questionnaires. The response rate in the age group 13-14 years was slightly higher at $84.4 \%$, with 10730 valid questionnaires.

The prevalence of rhinitis in the age group 6-7 years was: $29.4 \%$ "rhinitis ever", $24 \%$ recent rhinitis, $11.5 \%$ rhinoconjunctivitis and $0.1 \%$ severe rhinoconjunctivitis. In the group aged 13-14 the prevalence was $46.2 \%, 34.5 \%, 16.2 \%$ and $0.2 \%$, respectively (Table 1 ).

Table 1. Prevalence of rhinitis symptoms in children

\begin{tabular}{|c|c|c|c|c|}
\hline & \multicolumn{2}{|c|}{$6-7$ years } & \multicolumn{2}{|c|}{ 13-14 years } \\
\hline & $\mathrm{n}$ & $\%$ & $\mathrm{n}$ & $\%$ \\
\hline \multicolumn{5}{|c|}{ Rhinitis once } \\
\hline No & 7546 & 70.6 & 5775 & 53.8 \\
\hline Yes & 3144 & 29.4 & 4955 & 46.2 \\
\hline \multicolumn{5}{|c|}{ Recent $^{\mathrm{a}}$ rhinitis } \\
\hline No & 8128 & 76.0 & 7032 & 65.5 \\
\hline Yes & 2562 & 24.0 & 3698 & 34.5 \\
\hline \multicolumn{5}{|c|}{ Recent $^{\mathrm{a}}$ rhinoconjunctivitis } \\
\hline No & 9460 & 88.5 & 8993 & 83.8 \\
\hline Yes & 1230 & 11.5 & 1737 & 16.2 \\
\hline \multicolumn{5}{|c|}{ Severe rhinoconjunctivitis } \\
\hline No & 10680 & 99.9 & 10713 & 99.8 \\
\hline Yes & 10 & 0.1 & 17 & 0.2 \\
\hline
\end{tabular}

${ }^{\text {a }}$ The term Recent refers to the previous 12 mo.

Table 2 shows the main characteristics of children and the prevalence of risk factors (gender, parental smoking, weight, dog/cat in the home in the last 12 months, dog/cat in the home during the first year of life and maternal educational level). 
Table 2. Main characteristics of the children and prevalence of risk factors

\begin{tabular}{|c|c|c|c|c|}
\hline & \multicolumn{2}{|c|}{ 6-7 years } & \multicolumn{2}{|c|}{$13-14$ years } \\
\hline & $\mathrm{n}$ & $\%$ & $\mathrm{n}$ & $\%$ \\
\hline \multicolumn{5}{|l|}{ Gender } \\
\hline Female & 5270 & 49.8 & 5269 & 49.7 \\
\hline Male & 5321 & 50.2 & 5323 & 50.3 \\
\hline \multicolumn{5}{|l|}{ Parental smoking } \\
\hline Neither parent & 5020 & 48.7 & 5057 & 48.4 \\
\hline Father only & 1941 & 18.8 & 1893 & 18.1 \\
\hline Mother only & 1354 & 13.1 & 1487 & 14.2 \\
\hline Both parents & 1999 & 19.4 & 2016 & 19.3 \\
\hline \multicolumn{5}{|l|}{ Obesity } \\
\hline Normal weight & 5261 & 67 & 7421 & 82.3 \\
\hline Overweight & 1834 & 23.4 & 1396 & 15.5 \\
\hline Obese & 752 & 9.6 & 197 & 2.2 \\
\hline \multicolumn{5}{|l|}{ Cat at home in the past 12 months } \\
\hline Yes & 773 & 7.4 & 1722 & 16.2 \\
\hline No & 9699 & 92.6 & 8903 & 83.8 \\
\hline \multicolumn{5}{|l|}{ Cat at home in the first year of life } \\
\hline Yes & 584 & 5.5 & 879 & 11.6 \\
\hline No & 10026 & 94.5 & 6730 & 88.4 \\
\hline \multicolumn{5}{|l|}{ Dog at home in the past 12 months } \\
\hline Yes & 1313 & 12.6 & 3050 & 28.7 \\
\hline No & 9111 & 87.4 & 7563 & 71.3 \\
\hline \multicolumn{5}{|l|}{ Dog at home in the first year of life } \\
\hline Yes & 1123 & 10.6 & 1559 & 20.5 \\
\hline No & 9457 & 89.4 & 6060 & 79.5 \\
\hline \multicolumn{5}{|l|}{ Maternal educational level } \\
\hline No shooling/elementary & 2981 & 28.4 & 2261 & 22.1 \\
\hline High school & 4012 & 38.2 & 4418 & 43.2 \\
\hline University & 3519 & 33.5 & 3553 & 34.7 \\
\hline
\end{tabular}

With regard to domestic tobacco smoke exposure, in the age group 6-7 years this was 51.3\%; only the father smoked in $18.8 \%$ of cases, only the mother smoked in $13.1 \%$, both parents smoked in $19.4 \%$, and neither parent smoked in $48.7 \%$ of cases. In the age group of adolescents, exposure occurred in $51.6 \%$ of cases; in $18.1 \%$ of cases only the father smoked, in $14.2 \%$ only the mother smoked, in $19.3 \%$ both parents smoked and in $48.4 \%$ of cases neither parent smoked (Table 2).

On multivariate analysis, parental smoking was associated with a higher prevalence of forms of rhinitis in adolescents only when the mother was a smoker. The greatest effect was observed for "rhinitis ever" with an odds ratio of 1.20 (95\% confidence interval: 1.02-1.40) and "recent rhinitis" with an odds ratio of 1.19 (95\% confidence interval: 1.01-1.40) when the mother was a smoker (Table 3). In the group aged 6-7 years, the only significant relationship was found between parental smoking and "rhinitis ever" with an odds ratio of 1.19 (95\% confidence interval: 1.03-1.37) when both parents were smokers (Table 3). No significant relationships were found between the other forms of rhinitis and parental smoking. 
Table 3. Odds ratio for prevalence of rhinitis symptoms according to parental smoking in children and adolescents

\begin{tabular}{llll}
\hline & $\begin{array}{l}\text { Rhinitis ever } \\
\text { OR(95\% CI) }\end{array}$ & $\begin{array}{l}\text { Recent }^{\mathrm{a}} \text { rhinitis } \\
\text { OR }(95 \% \mathrm{CI})\end{array}$ & $\begin{array}{l}\text { Rhinoconjunctivitis } \\
\text { OR }(95 \% \mathrm{CI})\end{array}$ \\
\hline 6-7 years & & & \\
$\quad$ Neither parent & 1 & 1 & 1 \\
Father only & $1.08(0.94-1.25)$ & $1.07(0.92-1.24)$ & $1.09(0.89-1.32)$ \\
Mother only & $1.02(0.87-1.20)$ & $1.01(0.85-1.19)$ & $1.05(0.83-1.32)$ \\
Both parents & $1.19(1.03-1.37)$ & $1.15(0.99-1.33)$ & $1.19(0.97-1.45)$ \\
13-14 years & & & 1 \\
Neither parent & 1 & 1 & $0.90(0.74-1.10)$ \\
Father only & $1.05(0.92-1.22)$ & $0.94(0.80-1.09)$ & $1.20(0.97-1.48)$ \\
Mother only & $1.20(1.02-1.40)$ & $1.19(1.01-1.40)$ & $1.16(0.96-1.40)$ \\
Both parents & $1.14(0.99-1.32)$ & $1.05(0.90-1.22)$ & \\
& & &
\end{tabular}

\footnotetext{
a The term Recent refers to the previous $12 \mathrm{mo}$. CI, confidence interval; OR, odds ratio adjusted for gender, cat or dog in the home and maternal educational level.
}

The category "severe rhinoconjunctivitis" was not included in the regression analysis in either group due to low prevalence: $0.1 \%$ in the age group 6-7 years and $0.2 \%$ in the age group $13-14$ years.

\section{DISCUSSION}

The prevalence of "rhinitis ever" in our population ( $29.4 \%$ in children and $46.2 \%$ in adolescents) is similar to that reported by López-Silvarrey Varela et al. ${ }^{1}$ for a nearby population: $30.4 \%$ in children and $47.3 \%$ in adolescents.

On the Isle of Wight the prevalence of rhinitis in 10-year-old children was analysed using the same methodology as that of our study: it ranged from $22.6 \%$ in $1999-2000$ to $28.1 \%$ in the period 2011-2012. These figures are similar to those in our study, although the age groups studied are different. ${ }^{15}$

The older group had greater numbers of prevalence of rhinitis, would this not be expected given that they have had several more years to develop rhinitis. Our results agree with those of other geographical areas as indicated in various populations; Asher et al. ${ }^{16}$ using the same methodology as in our study, reported the prevalence of rhinoconjunctivitis in children aged 6-7 years and 13-14 years in different countries and regions. For the age group 13-14 years in Spain, the prevalence was 15\%, slightly lower than the $16.5 \%$ observed in our study. Similar rates were observed in countries such as Italy (15.5\%) and Germany (15\%), but the prevalence was lower in other European countries such as Portugal (9.5\%). For the age group 6-7 years, the prevalence of rhinoconjunctivitis was 7.9\% in Spain, 6.5\% in Italy, 6.9\% in Germany and $9.3 \%$ in Portugal, all lower than the $11.5 \%$ found in our study $(11.5 \%)$.

Ait-Khaled et al. ${ }^{13}$ reported the prevalence of different types of rhinitis in children aged 13-14 years, like that of the adolescents in our study, in many countries around the world. In neighbouring countries, the prevalence of "rhinitis ever" was $26.5 \%$ in Portugal (range: $21.4 \%-31.7 \%$ according to geographic region) and 31\% in Italy (range: $15.1 \%-38.4 \%$ according to geographic region), which are lower than the $46.2 \%$ found for "rhinitis ever" in our group of adolescents.

In our study we did not find an elevated exposure to cats and dogs; however, these results are similar to other publications in our country. ${ }^{17}$ 
Another finding of the present study was that exposure to parental smoking remained high in this community (about $51 \%$ in both age groups), although a downward trend was observed with respect to the results of a previous study conducted between 1990 and 1992 with six to 18-year-olds in the same community, where the prevalence of parental smoking was $57 \%$ in males and $55 \%$ in female women. ${ }^{18}$

Regarding the relationship between exposure to parental smoking and rhinitis, we found an increased prevalence of symptoms of rhinitis ("rhinitis ever") when both parents were smokers in the group of children aged 6-7 years. In the group of adolescents aged 13-14 years, this was found only when the mother was a smoker for both "rhinitis ever" and recent rhinitis.

In a study conducted in Stockholm, Thatcher et al. showed that exposure to tobacco smoke during childhood was associated with an overall increased risk of rhinitis (odds ratio: 1.22; 95\% confidence interval: 1.022-1.46) at the age of 16 years, although the method used to measure exposure to tobacco smoke was different from that of our study. When specific associations were examined by age, the increase in risk associated with exposure to secondhand smoke in the womb or during childhood was mostly confined to early childhood. ${ }^{8}$

In a study performed in Hong Kong, Lee et al. analysed the effect of exposure to active and passive maternal smoking during pregnancy. They reported that foetal exposure to passive maternal smoking was significantly associated with allergic rhinitis (odds ratio: 1.22, 95\% confidence interval: 1.09-1.37), but active maternal smoking was not significantly associated with allergic rhinitis (odds ratio: 1.01, 95\% confidence interval: $0.70-1.46){ }^{10}$

In the work by Mitchell et al., ${ }^{11}$ the authors confirmed the importance of maternal smoking, and the separate and additional effect of paternal smoking with a dose-response effect on asthma symptoms suggesting a causal relationship, but for the rhinoconjunctivitis the causal relationship was less certain. The work of Montfort and colleagues goes further; it revealed a protective effect of exposure to tobacco smoke when both parents smoked against "rhinitis ever" (odds ratio: $0.82(0.66-1) P<.01)$ and recent rhinitis (odds ratio: $0.66(0.5-0.88) P<.001) .{ }^{12}$ These findings may be related to the "healthy smoker" phenomenon rather than a real protective effect of exposure to tobacco smoke. When it is find a protective effect of smoking on the prevalence or severity of rhinitis, rather than real protection due to smoking, this result would be because people with fewer symptoms continue smoking and people with more symptoms quit smoking or no longer be initiated into this habit. ${ }^{19}$

Several pathophysiological mechanisms seem to support the harmful effect of ETS on the respiratory system of children. Foetal exposure to tobacco smoke during pregnancy may occur because nicotine and other toxic substances readily cross the placental barrier. ${ }^{20}$ In addition, mothers who smoke are often not able to quit during pregnancy. ${ }^{21}$ Smoking during pregnancy is known to affect foetal development, maturation of the lungs and the pulmonary immune system, favouring prematurity and intrauterine growth retardation, leading to a lower calibre airways.

Infants and pre-school children are most vulnerable to the effects of ETS exposure, as they have a lower body mass and their active anabolic state means they have a higher respiratory rate and increased ventilation per minute per unit of body mass at rest, and consequently they inhale a greater effective dose of pollutants per $\mathrm{kg}$ body weight. ${ }^{22}$ This represents direct toxicity for the airways, oxidative damage, inflammatory cell recruitment, increased neutrophilic inflammation, increased epithelial permeability, disposition to respiratory infections, allergic sensitisation, poor response to treatment with corticosteroids and changes in the cytokine profile. ${ }^{23}$

The fact that the significant effect mainly relates to maternal smoking seems reasonable, given the possible effect, already mentioned, of tobacco smoke on the airways during pregnancy. ${ }^{20,21}$ Moreover, children usually spend more time with their mothers than their fathers, so maternal smoking has a greater impact on their health than paternal smoking. ${ }^{24}$ 
The present study has certain limitations. The cross-sectional design prevents establishing any causal relationship between parental smoking and rhinitis symptoms. In addition, the data were obtained from a questionnaire and there was no objective measurement of exposure to tobacco smoke. However, the validity of questionnaires to assess smoking status in epidemiological studies is widely accepted. ${ }^{25}$ The strengths of the study are the large sample size, the random selection of children and adolescents included, and the use of the thoroughly validated ISAAC study methodology.

In conclusion, rhinitis is highly prevalent in our community. Exposure to ETS by children and adolescents is still very common despite having decreased slightly in recent years. The relationship between ETS and rhinitis symptoms in children and adolescents of this community is not as robust as that found for asthma.

\section{Conflict of interest}

None.

\section{References}

1. López-Silvarrey Varela A, González Barcala FJ, Paz Esquete JJ, Pérez Castro TR, Valdés Cuadrado L, Castro Iglesias A. Prevalencia de síntomas de asma y rinitis en la población de A Coruña. An Pediatr. 2007;66:146-153.

2. Braback L, Hjern A, Rasmussen F. Trends in asthma, allergic rhinitis and eczema among Swedish conscripts from farming and non-farming environments. A nationwide study over three decades. Clin Exp Allergy. 2004;34:38-43.

3. Lee SL, Wong W, Lau YL. Increasing prevalence of allergic rhinitis but not asthma among children in Hong Kong from 1995 to 2001 (Phase 3 International Study of Asthma and Allergies in Childhood). Pediatr Allergy Immunol. 2004;15:72-78.

4. Nolte H, Nepper-Christensen S, Backer V. Unawareness and undertreatment of asthma and allergic rhinitis in a general population. Respir Med. 2006;100:354-362.

5. Belushkin M, Jaccard G, Kondylis A. Considerations for comparative tobacco product assessments based on smoke constituent yields. Regul Toxicol Pharmacol. 2015;73:105-113.

6. Cheraghi M, Salvi S. Environmental tobacco smoke (ETS) and respiratory health in children. Eur J Pediatr. 2009;168:897-905.

7. Oberg M, Jaakkola MS, Woodward A, Peruga A, Prüss-Ustün A. Worldwide burden of disease from exposure to second-hand smoke: a retrospective analysis of data from 192 countries. Lancet. 2011;377:139-146.

8. Thacher JD, Gruzieva O, Pershagen G, et al. Pre- and postnatal exposure to parental smoking and allergic disease through adolescence. Pediatrics. 2014;134:428-434.

9. Gonzalez-Barcala FJ, Pertega S, Sampedro M, et al. Impact of parental smoking on childhood asthma. J Pediatr (Rio J). 2013;89:294-299.

10. Lee SL, Lam TH, Leung TH, et al. Foetal exposure to maternal passive smoking is associated with childhood asthma, allergic rhinitis, and eczema. Sci World J. 2012;2012:542983

11. Mitchell EA, Beasley R, Keil U, et al. The association between tobacco and the risk of asthma, rhinoconjunctivitis and eczema in children and adolescents: analyses from Phase Three of the ISAAC programme. Thorax. 2012;67:941-949.

12. Montefort S, Ellul P, Montefort M, Caruana S, Grech V, Agius Muscat H. The effect of cigarette smoking on allergic conditions in Maltese children (ISAAC). Pediatr Allergy Immunol. 2012;23:472-478.

13. Aït-Khaled N, Pearce N, Anderson HR, Ellwood P, Montefort S, Shah J, ISAAC Phase Three Study Group. Global map of the prevalence of symptoms of rhinoconjunctivitis in children: the International Study of Asthma and Allergies in Childhood (ISAAC) Phase Three. Allergy. 2009;64:123-148.

14. Cole TJ, Bellizzi MC, Flegal KM, Dietz WH. Establishing a standard definition for child overweight and obesity worldwide: international survey. BMJ. 2000;320:1240-1243. 
15. Patil VK, Kurukulaaratchy RJ, Venter C, et al. Changing prevalence of wheeze, rhinitis and allergic sensitisation in late childhood: findings from 2 Isle of Wight birth cohorts' 12-years apart. Clin Exp Allergy. 2015;45:1430-1438.

16. Asher MI, Montefort S, Björkstén B, et al. Worldwide time trends in the prevalence of symptoms of asthma, allergic rhinoconjunctivitis, and eczema in childhood: ISAAC phases one and three repeat multicountry cross-sectional surveys. Lancet. 2006;368:733-743.

17. Arnedo-Pena A, García-Marcos L, García Hernández G, et al. Time trends and geographical variations in the prevalence of symptoms of allergic rhinitis in 6-7-year-old children from eight areas of Spain according to the ISAAC. An Pediatr. 2005;62:229-236.

18. González Barcala FJ, Takkouche B, Valdés L, et al. Parental smoking and lung function in healthy children and adolescents. Arch Bronconeumol. 2007;43:81-85.

19. Becklake MR, Lalloo U. The 'healthy smoker': a phenomenon of health selection? Respiration. 1990;57:137-144.

20. Wu FY, Chiu HT, Wu HD, Lin CJ, Lai JS, Kuo HW. Comparison of urinary and plasma cotinine levels during the three trimesters of pregnancy. Paediatr Perinat Epidemiol. 2008;22:296-301.

21. Ebrahim SH, Floyd RL, Merritt RK 2nd, Decoufle P, Holtzman D. Trends in pregnancy-related smoking rates in the United States, 1987-1996. JAMA. 2000;283:361-366.

22. Abu-Baker NN, Haddad L, Savage C. The influence of secondhand smoke exposure on birth outcomes in Jordan. Int J Environ Res Public Health. 2002;7:616-634.

23. Nakamura Y, Miyata M, Ohba T, et al. Cigarette smoke extract induces thymic stromal lymphopoietin expression, leading to $\mathrm{T}(\mathrm{H}) 2$-type immune responses and airway inflammation. $J$ Allergy Clin Immunol. 2008;122:1208-1214.

24. Offer S. Time with children and employed parents' emotional well-being. Soc Sci Res. 2014;47:192-203.

25. Gehring U, Leaderer BP, Heinrich J, et al. Comparison of parental reports of smoking and residential air nicotine concentrations in children. Occup Environ Med. 2006;63:766-772. 